\title{
SARS-CoV-2 VOCs immune evasion from previously elicited neutralizing antibodies is mainly driven by lower cross-reactivity due to Spike RBD electrostatic surface changes
}

Matheus V. F. Ferraz ${ }^{a, b}$, Emerson G. Moreira ${ }^{a, b}$, Danilo F. Coêlho ${ }^{a, b}$, Gabriel Luz Wallau and Roberto D. Lins ${ }^{a,{ }^{*}}$ on behalf of Fiocruz COVID-19 Genomic Surveillance Network

${ }^{a}$ Aggeu Magalhães Institute, Oswaldo Cruz Foundation, Recife, PE, Brazil

${ }^{b}$ Department of Fundamental Chemistry, Federal University of Pernambuco, Recife, PE, Brazil

*Corresponding author: roberto.lins@cpqam.fiocruz.br

SARS-CoV-2 VOCs immune evasion is mainly due to lower cross-reactivity from previously elicited antibodies, while increased affinity to hACE2 plays a minor role. Low affinity between those antibodies and SARS-CoV-2 VOCs are caused by remodeling in the electrostatic surface potential of the Spike RBDs. P.3 variant is a putative VOC.

\section{Introduction}

The COVID-19 pandemic has dramatically impacted the world population since 2019 and currently accounts for more than 2 million deaths. ${ }^{1}$ The genome evolution of its etiological agent, the severe acute respiratory syndrome coronavirus 2 (SARS-CoV-2), has been closely monitored since the rapid sharing of the first genomic sequences in December $2019 .^{2}$ SARS-CoV-2 presents a relatively low mutation rate compared to other RNA viruses, ${ }^{3}$ and hence few genomic sites accumulated mutations and were fixed until the second quarter of 2020. ${ }^{4}$ However, a substantially different scenario emerged between September-December 2020 with the detection of independent variants of concern (VOCs - B.1.1.7, ${ }^{5}$ B.1.351, ${ }^{6}$ and P. $1^{7}$ ) lineages bearing multiple amino acid replacements (K417T, E484K, and N501Y) and indels in the Spike protein, ${ }^{8}$ in which some researchers hypothesized to have occurred due to a "global shift in the 
SARS-CoV-2 selective landscape". ${ }^{9}$ Although large-scale immunological studies are not available so far, the main hypothesis to explain such a global shift takes into account the rising population immunity, which would naturally select escape mutants with a higher fitness compared to previous circulating lineages. To support this hypothesis, some evidence could be mentioned, such as the increasing number of reinfection cases with VOCs and variants of interest (VOI) carrying some of the same amino acid mutation (E484K), ${ }^{10}$ the continuum emergence of new VOIs carrying E484K and N501Y during the first months of $2021^{11}$ and the recurrent emergence of some of those Spike amino acid changes in SARS-CoV-2 experimental evolution settings challenged with monoclonal and polyclonal antibodies. ${ }^{12}$

To enter the host cell, SARS-CoV-2 makes use of the glycoprotein Spike (S). Protein $\mathrm{S}$ is a homotrimer and each monomer has two subunits, S1 and S2. The S1 subunit contains the receptor-binding domain (RBD), which binds to the human receptor angiotensin-converting enzyme 2 (hACE2), thus allowing the fusion of membranes and entry into the cell. ${ }^{13}$ Among the 29 SARS-CoV- 2 encoded proteins, the S protein has been investigated more thoroughly due to its key role in hACE2 binding, and because the RBD region is one of the main targets of neutralizing antibodies ( $\mathrm{nAbs}$ ) produced from the human immunological response against the SARS-CoV-2. ${ }^{14}$ Deep mutational scanning of the RBD region has identified that most amino acid changes are deleterious for hACE2 binding, whereas a few marginally enhance the affinity to hACE2, ${ }^{15}$ including some that have been detected in VOCs, such as N501Y in the more transmissible and mortal B.1.1.7 lineage. ${ }^{16}$ On the other hand, amino acid changes such as K417N, E484K, and N501Y found in VOCs P. $1^{17}$ and B.1.35 $1^{8}$ have been shown to increase viral fitness by lowering the effectiveness of neutralizing monoclonal and/or polyclonal antibodies. ${ }^{11}$ Therefore, the emergence and spread of more fit VOCs lineages may be driven by a more complex mechanism other than the often-proposed affinity increase between hACE2 and SARS-CoV-2 Spike protein.

\section{Results and Discussion}

Despite the extensive description of mutations occurring in the SARS-CoV-2 RBD, little is known about their impact on receptor recognition, namely hACE2. In this regard, Starr et al15 
have systematically measured the impact of every amino acid in the RBD, by replacing for the 20 amino acids in each position, towards hACE2 binding affinity, expressed as the $\Delta \log \left(\mathrm{K}_{\mathrm{D}}\right)$, in which $K_{D}$ represents the dissociation constant. Changes in the $K_{D}$ upon single-point mutations were obtained from a deep mutational scanning library using the yeast-surface display technique. We have converted the $\Delta \log \left(\mathrm{K}_{\mathrm{D}}\right)$ into the change in the mutational Gibbs free energy of binding $\Delta \Delta G$ for all sites of interaction between SARS-CoV-2 RBD and hACE2 (more details in Electronic Supplementary Information) and observed that whereas most of the single mutations on the RBD tend to be deleterious (i.e, $\Delta \Delta G>0$ ), none of the mutations would dramatically enhance the affinity for the hACE2 (Figure S1). Since the wild-type (WT) RBD (from Wuhan reference genome) binds the hACE2 in the nanomolar range, variations in the $K_{D}$ on the order of $1 \mathrm{E}+1$, resulting in approximately $0.5-1.5 \mathrm{kcal}^{\mathrm{mol}}{ }^{-1}$, do not have the potential to significantly impact the binding affinity. However, it is important to stress that deep mutational scanning binding $\Delta \Delta G$ values were reported for single mutations only and the novel SARS-CoV-2 VOCs often involve more than one mutation on the RBD.

Aiming to investigate whether multiple mutations could lead to a significant increase in affinity to hACE2, variation in binding free energy calculations were performed between the RBDs of selected VOCs and hACE2. For 4 out of 7 lineages evaluated, the binding $\Delta \Delta G$ values fell within the mean absolute error (MAE) associated with the method used (ca. $\pm 1.7 \mathrm{REU}$, by testing the $\Delta \Delta G$ calculations considering the 4,000 mutations from the deep mutational scanning data, i.e, a dataset containing a similar system to the calculations we have performed; see methodology section of the ESI), whereas the remaining values have positive values and are boardliners to the error limit (Figure 1B). This finding is in agreement with data from the above discussed deep mutational scanning experiments ${ }^{15}$ and bio-layer interferometry assays, ${ }^{18}$ where all measurements have shown $\mathrm{K}_{\mathrm{D}}$ values for different RBDs to hACE2 within $10^{-8}$ to $10^{-9} \mathrm{M}$. Therefore, assumptions that higher transmissibility is mainly associated with mutations in the RBD that leads to an enhanced affinity to hACE2 must be revisited, as both experiments and calculations do not show substantial evidence corroborating this hypothesis.

On the other hand, a plethora of studies has demonstrated antibody evasion for some of the novel VOCs. ${ }^{19-28}$ However, data correlating immune evasion of SARS-CoV-2 VOCs with binding free energies are currently not available. In order to evaluate the thermodynamics contribution of such low cross-reactivity, we calculated the variation in binding free energy 
values for a selected set of SARS-CoV-2 VOCs RBDs complexed to 21 known nAbs for which atomic coordinates were made publicly available (Table S2). These nAbs bind to two distinct regions of the viral RBD. As illustrated in Figure 1A, all nAbs block the RBD access to hACE2; however, classes I and II have a higher spatial overlap with the hACE2 binding site compared to classes III and IV. ${ }^{29}$ The variations in binding free energies are shown in Figure 1B. The data suggest that binding is highly compromised between most nAbs elicited against RBD of previous circulating non-VOCs lineages when compared to VOCs RBD, with the only exception being lineage B.1.429. It is important to note that this variant was identified in Portugal and California (USA) $)^{30}$ at a similar time frame as VOCs B.1.1.7, B.1.351, and P.1, but it has not spread and increased in frequency significantly as recognized VOCs did. Interestingly, the calculated variations in binding free energies is able to capture the trend, i.e., variants that emerged and rapidly reached higher frequencies outcompeting other circulating lineages (VOCs) by having a larger number of nAbs that would not bind to the VOCs. Our calculations also corroborate with available experimental data for nAbs in our dataset. nAbs STE90-C11, ${ }^{31}$ and $\mathrm{C}_{102}{ }^{29}$ were verified to continue to bind to RBD containing the E484K mutation (lineage P.2). The calculated $\Delta \Delta G$ for these nAbs against P.2 are within the MAE (Table S2). On the other hand, nAb 15033 moderately loses its neutralization power against a pseudo-virus containing the E484K mutation, ${ }^{32}$ a finding supported by a positive $\Delta \Delta G$ variation above the MAE (Table S2). As for nAb BD-629, a neutralization assay was performed with the pseudovirus containing the L452R mutation, ${ }^{33}$ which corresponds to lineage B.1.429. It showed that even after the mutation, the nAb continues to neutralize the virus, which is also corroborated by our calculations (Table S2). Moreover, our data also suggest that variants B.1.526 and P.2 would have limited spread as a large number of nAbs are still capable of binding to their RBD and, hence neutralizing it. Conversely, most of the tested nAbs are expected not to bind efficiently to P.3 RBD, a recently reported lineage detected in the Philippines, ${ }^{34}$ similar to recognized VOCs B.1.1.7, B.1.351 and P.1, suggesting that this variant is a putative VOC with a high spreading potential in a population with medium to high community immunity against previous circulating lineages due to immune evasion. (We would like to note that similar results were obtained when using FoldX ${ }^{35}$ (Figure S2), a different software package/algorithm, in which its empirical energy function provides an MAE of $0.46 \mathrm{kcal}^{\mathrm{mol}}{ }^{-1}$, 36 therefore providing further support to these observations). 
Mutations on the epitope may lead to disruptions and/or change of specific interactions with its respective antibody. However, the energy loss associated with that single or a few specific interactions may not account for the large energy variation shown in Figure 1B (even taking into account the associated error of the method). Therefore, to gain insight into the molecular mechanism behind low nAbs crossreactivity to the VOCs RBD, we have calculated the electrostatic potential of each RBD and plotted onto their molecular surface (Figure 1C). The surface charge profile of lineage B.1.429 is remarkably similar to the reference (Wuhan) lineage, suggesting that existing nAbs are able to bind to its RBD. In contrast, significant changes in surface charge profile can be seen for the higher spreading variants, especially B.1.351 and P.1. Therefore, in addition to loss/change of specific interactions, change in global electrostatics will affect overall charge complementary between current nAbs and VOCs RBD.

\section{Conclusions}

In this study, leveraging structural data and computer modelling techniques, we investigated the variation in the binding free-energy $(\Delta \Delta G)$ profile of VOCs and VOIs SARS-CoV-2 lineages with hACE2 and with a dataset of known human nAbs. In agreement with the available experimental data, our results show only a marginal impact of VOC RBD amino acid changes to hACE2 affinity. On the other hand, we found that VOCs RBDs have a significant unfavorable $\Delta \Delta G$ to nAbs that can be related to changes in the electrostatic potential surface profiles, hence identifying the molecular and thermodynamical components behind SARS-CoV-2 antibody evasion. In addition, our data suggests that a close attention should be given to lineage P.3, as it likely holds a high spreading potential in a human population with rising immunity. In summary, the current observed higher transmission of SARS-CoV-2 VOCs is likely associated with a partial or complete failure of the antibody recognition and neutralization in individuals previously exposed to SARS-CoV-2 non-VOC variants. These results have key implications on i. the basic understanding of VOCs emergence and maintenance;

ii. on the rational design of antibody-based therapeutics; iii. vaccine efficacy and updates; and iv. may be exploited to rapidly screen immune scape worrisome lineages. 


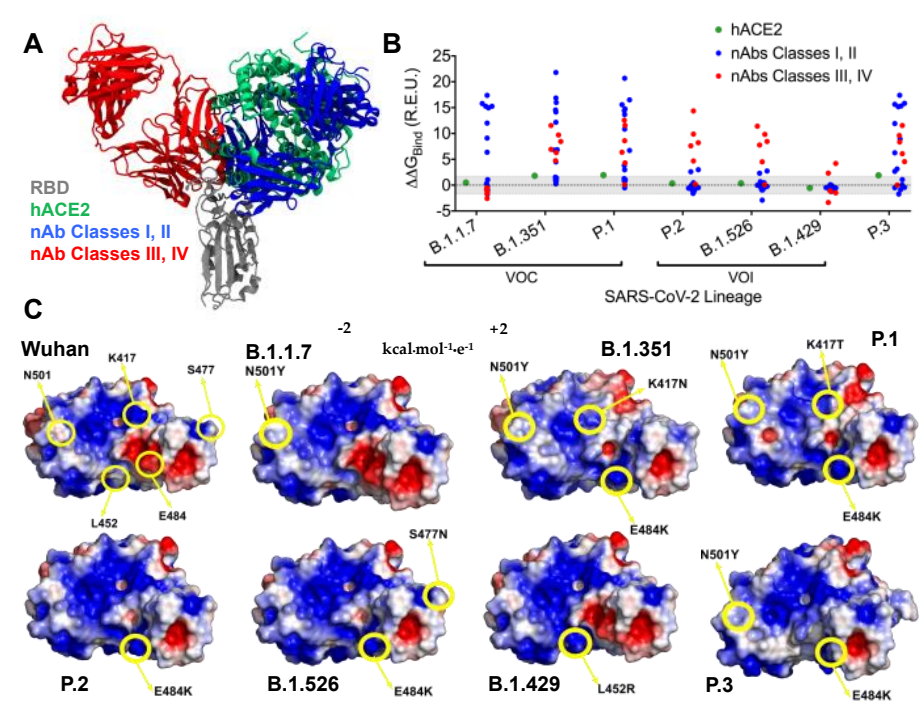

Figure 1. Title. A) Cartoon representation of the SARS-CoV-2 RBD (gray) bound to hACE2 (green) and representative nAbs from classes I and II (blue) and II and IV (red); B) Calculated relative binding free energies of RBDs complexed to hACE and nAbs (RBD from Wuhan lineage is taken as reference); C) Electrostatic surface potential of SARSCoV-2 RBD reference and selected variants.

RDL and GW designed the study. MVFF, EGM and DFC performed all modeling, calculations and data analysis. The manuscript was written with the input from all authors.

The authors have declared no competing interests. We thank all the health care workers and scientists who have worked hard to deal with this pandemic threat, the GISAID team, and all the EpiCoV database's submitters, GISAID acknowledgment table containing sequences used in this study are attached to this post (Table S1). This study was supported by grants from FACEPE, CAPES, CNPq and INCT-FCX and FIOCRUZ COVID-19 Genomic Surveillance Network. Computer allocation was partly granted by the Brazilian National Scientific Computing Center (LNCC). GLW and RDL are supported by the CNPq through their productivity research fellowships (303902/2019-1 and 425997/2018-9).

\section{Notes and References}

1. E. Dong, H. Du and L. Gardner, Lancet Infect Dis, 2020, 20, 533-534. 
2. P. Zhou, X. L. Yang, X. G. Wang, B. Hu, L. Zhang, W. Zhang, H. R. Si, Y. Zhu, B. Li, C. L. Huang, H. D. Chen, J. Chen, Y. Luo, H. Guo, R. D. Jiang, M. Q. Liu, Y. Chen, X. R. Shen, X. Wang, X. S. Zheng, K. Zhao, Q. J. Chen, F. Deng, L. L. Liu, B. Yan, F. X. Zhan, Y. Y. Wang, G. F. Xiao and Z. L. Shi, Nature, 2020, 579, 270-273.

3. L. van Dorp, M. Acman, D. Richard, L. P. Shaw, C. E. Ford, L. Ormond, C. J. Owen, J. Pang, C. C. S. Tan, F. A. T. Boshier, A. T. Ortiz and F. Balloux, Infect Genet Evol, 2020, 83, 104351.

4. D. Mercatelli and F. M. Giorgi, Front Microbiol, 2020, 11, 1800.

5. A. Rambaut, N. Loman, O. Pybus, W. Barclay, J. Barrett, A. Carabelli, T. Connor, T. Peacock, D. L. Robertson and E. Volz, Genom. Epidemiol, 2020.

6. H. Tegally, E. Wilkinson, M. Giovanetti, A. Iranzadeh, V. Fonseca, J. Giandhari, D. Doolabh, S. Pillay, E. J. San, N. Msomi, K. Mlisana, A. von Gottberg, S. Walaza, M. Allam, A. Ismail, T. Mohale, A. J. Glass, S. Engelbrecht, G. Van Zyl, W. Preiser, F. Petruccione, A. Sigal, D. Hardie, G. Marais, M. Hsiao, S. Korsman, M.-A. Davies, L. Tyers, I. Mudau, D. York, C. Maslo, D. Goedhals, S. Abrahams, O. Laguda-Akingba, A. Alisoltani-Dehkordi, A. Godzik, C. K. Wibmer, B. T. Sewell, J. Lourenço, L. C. J. Alcantara, S. L. K. Pond, S. Weaver, D. Martin, R. J. Lessells, J. N. Bhiman, C. Williamson and T. de Oliveira, medRxiv, 2020, DOI: 10.1101/2020.12.21.20248640, 2020.2012.2021.20248640.

7. N. R. Faria, I. M. Claro, D. Candido, L. A. Moyses Franco, P. S. Andrade, T. M. Coletti, C. A. M. Silva, F. C. Sales, E. R. Manuli and R. S. Aguiar, Virological. org, 2021, 586.

8. H. Tegally, E. Wilkinson, M. Giovanetti, A. Iranzadeh, V. Fonseca, J. Giandhari, D. Doolabh, S. Pillay, E. J. San, N. Msomi, K. Mlisana, A. von Gottberg, S. Walaza, M. Allam, A. Ismail, T. Mohale, A. J. Glass, S. Engelbrecht, G. Van Zyl, W. Preiser, F. Petruccione, A. Sigal, D. Hardie, G. Marais, M. Hsiao, S. Korsman, M. A. Davies, L. Tyers, I. Mudau, D. York, C. Maslo, D. Goedhals, S. Abrahams, O. Laguda-Akingba, A. Alisoltani-Dehkordi, A. Godzik, C. K. Wibmer, B. T. Sewell, J. Lourenco, L. C. J. Alcantara, S. L. Kosakovsky Pond, S. Weaver, D. Martin, R. J. Lessells, J. N. Bhiman, C. Williamson and T. de Oliveira, Nature, 2021, DOI: 10.1038/s41586-021-03402-9.

9. D. P. Martin, S. Weaver, H. Tegally, E. J. San, S. D. Shank, E. Wilkinson, J. Giandhari, S. Naidoo, Y. Pillay, L. Singh, R. J. Lessells, S. A. Ngs, C.-G. UK, R. K. Gupta, J. O.

Wertheim, A. Nekturenko, B. Murrell, G. W. Harkins, P. Lemey, O. A. MacLean, D. L. Robertson, T. de Oliveira and S. L. K. Pond, medRxiv, 2021, DOI:

10.1101/2021.02.23.21252268.

10. N. Felipe, C. Cristiano da, N. Valdinete, S. Victor, C. André, N. Fernanda, C. Ágatha, D. Débora, S. George, M. Matilde, P. Karina, G. Luciana, B. Maria Júlia, J. Michele, S. Marineide, M. Tirza, A. Lígia, S. João Hugo, C.-F. Rubens, S. Tsuyoshi, I. Kentaro, H. Masanori, K. Makoto, S. Marilda Mendonça, W. Gabriel Luz, D. Edson, G. Tiago, B. Gonzalo and R. Paola Cristina, Research Square, 2021, DOI: 10.21203/rs.3.rs318392/v1.

11. A. J. Greaney, A. N. Loes, K. H. D. Crawford, T. N. Starr, K. D. Malone, H. Y. Chu and J. D. Bloom, Cell Host Microbe, 2021, 29, 463-476 e466.

12. Z. Wang, F. Schmidt, Y. Weisblum, F. Muecksch, C. O. Barnes, S. Finkin, D. SchaeferBabajew, M. Cipolla, C. Gaebler, J. A. Lieberman, T. Y. Oliveira, Z. Yang, M. E. Abernathy, K. E. Huey-Tubman, A. Hurley, M. Turroja, K. A. West, K. Gordon, K. G. Millard, V. Ramos, J. Da Silva, J. Xu, R. A. Colbert, R. Patel, J. Dizon, C. Unson-O'Brien, I. Shimeliovich, A. Gazumyan, M. Caskey, P. J. Bjorkman, R. Casellas, T. Hatziioannou, 
P. D. Bieniasz and M. C. Nussenzweig, Nature, 2021, DOI: 10.1038/s41586-02103324-6.

13. X. Chi, R. Yan, J. Zhang, G. Zhang, Y. Zhang, M. Hao, Z. Zhang, P. Fan, Y. Dong, Y. Yang,

Z. Chen, Y. Guo, J. Zhang, Y. Li, X. Song, Y. Chen, L. Xia, L. Fu, L. Hou, J. Xu, C. Yu, J. Li, Q. Zhou and W. Chen, Science, 2020, 369, 650-655.

14. M. McCallum, A. De Marco, F. A. Lempp, M. A. Tortorici, D. Pinto, A. C. Walls, M. Beltramello, A. Chen, Z. Liu, F. Zatta, S. Zepeda, J. di lulio, J. E. Bowen, M. MontielRuiz, J. Zhou, L. E. Rosen, S. Bianchi, B. Guarino, C. S. Fregni, R. Abdelnabi, S. C. Foo, P. W. Rothlauf, L. M. Bloyet, F. Benigni, E. Cameroni, J. Neyts, A. Riva, G. Snell, A. Telenti, S. P. J. Whelan, H. W. Virgin, D. Corti, M. S. Pizzuto and D. Veesler, Cell, 2021, DOI: 10.1016/j.cell.2021.03.028.

15. T. N. Starr, A. J. Greaney, S. K. Hilton, D. Ellis, K. H. D. Crawford, A. S. Dingens, M. J. Navarro, J. E. Bowen, M. A. Tortorici, A. C. Walls, N. P. King, D. Veesler and J. D. Bloom, Cell, 2020, 182, 1295-1310 e1220.

16. N. G. Davies, C. I. Jarvis, C. C.-W. Group, W. J. Edmunds, N. P. Jewell, K. Diaz-Ordaz and R. H. Keogh, Nature, 2021, DOI: 10.1038/s41586-021-03426-1.

17. N. Felipe, N. Valdinete, S. Victor, C. André, N. Fernanda, S. George, C. Ágatha, D. Débora, P. Karina, M. Matilde, B. Maria, J. Michele, G. Luciana, C. Cristiano da, S. Vanderson, B. Daniel, S. Marineide, M. Tirza, P. Gemilson, A. Ligia, S. João, A. Ighor, D. Filipe, S. Marilda, W. Gabriel, R. Paola, D. Edson, G. Tiago and B. Gonzalo, Nature Portfolio, 2021, DOI: 10.21203/rs.3.rs-275494/v1.

18. X. Zhu, D. Mannar, S. S. Srivastava, A. M. Berezuk, J.-P. Demers, J. W. Saville, K. Leopold, W. Li, D. S. Dimitrov, K. S. Tuttle, S. Zhou, S. Chittori and S. Subramaniam, bioRxiv, 2021, DOI: 10.1101/2021.01.11.426269, 2021.2001.2011.426269.

19. D. M. Altmann, R. J. Boyton and R. Beale, Science, 2021, 371, 1103-1104.

20. D. Zhou, W. Dejnirattisai, P. Supasa, C. Liu, A. J. Mentzer, H. M. Ginn, Y. Zhao, H. M.

E. Duyvesteyn, A. Tuekprakhon, R. Nutalai, B. Wang, G. C. Paesen, C. Lopez-Camacho, J. Slon-Campos, B. Hallis, N. Coombes, K. Bewley, S. Charlton, T. S. Walter, D. Skelly, S. F. Lumley, C. Dold, R. Levin, T. Dong, A. J. Pollard, J. C. Knight, D. Crook, T. Lambe, E. Clutterbuck, S. Bibi, A. Flaxman, M. Bittaye, S. Belij-Rammerstorfer, S. Gilbert, W. James, M. W. Carroll, P. Klenerman, E. Barnes, S. J. Dunachie, E. E. Fry, J.

Mongkolsapaya, J. Ren, D. I. Stuart and G. R. Screaton, Cell, 2021, DOI: 10.1016/j.cell.2021.02.037.

21. R. Wang, Q. Zhang, J. Ge, W. Ren, R. Zhang, J. Lan, B. Ju, B. Su, F. Yu, P. Chen, H. Liao, Y. Feng, X. Li, X. Shi, Z. Zhang, F. Zhang, Q. Ding, T. Zhang, X. Wang and L. Zhang, bioRxiv, 2021, DOI: 10.1101/2021.03.09.434497, 2021.2003.2009.434497.

22. C. K. Wibmer, F. Ayres, T. Hermanus, M. Madzivhandila, P. Kgagudi, B. Oosthuysen, B. E. Lambson, T. de Oliveira, M. Vermeulen, K. van der Berg, T. Rossouw, M. Boswell, V. Ueckermann, S. Meiring, A. von Gottberg, C. Cohen, L. Morris, J. N. Bhiman and P. L. Moore, Nat Med, 2021, DOI: 10.1038/s41591-021-01285-x.

23. W. F. Garcia-Beltran, E. C. Lam, K. St Denis, A. D. Nitido, Z. H. Garcia, B. M. Hauser, J. Feldman, M. N. Pavlovic, D. J. Gregory, M. C. Poznansky, A. Sigal, A. G. Schmidt, A. J. lafrate, V. Naranbhai and A. B. Balazs, Cell, 2021, DOI: 10.1016/j.cell.2021.03.013.

24. D. Focosi and F. Maggi, Rev Med Virol, 2021, DOI: 10.1002/rmv.2231.

25. C. Rees-Spear, L. Muir, S. A. Griffith, J. Heaney, Y. Aldon, J. L. Snitselaar, P. Thomas, C. Graham, J. Seow, N. Lee, A. Rosa, C. Roustan, C. F. Houlihan, R. W. Sanders, R. Gupta, 
P. Cherepanov, H. Stauss, E. Nastouli, K. J. Doores, M. J. van Gils and L. E. McCoy, bioRxiv, 2021, DOI: 10.1101/2021.01.15.426849, 2021.2001.2015.426849.

26. T. N. Starr, A. J. Greaney, A. Addetia, W. W. Hannon, M. C. Choudhary, A. S. Dingens, J. Z. Li and J. D. Bloom, Science, 2021, 371, 850.

27. A. J. Greaney, T. N. Starr, P. Gilchuk, S. J. Zost, E. Binshtein, A. N. Loes, S. K. Hilton, J. Huddleston, R. Eguia, K. H. D. Crawford, A. S. Dingens, R. S. Nargi, R. E. Sutton, N. Suryadevara, P. W. Rothlauf, Z. Liu, S. P. J. Whelan, R. H. Carnahan, J. E. Crowe and J. D. Bloom, Cell Host \& Microbe, 2021, 29, 44-57.e49.

28. B. Luan and T. Huynh, bioRxiv, 2021, DOI: 10.1101/2021.02.06.430088, 2021.2002.2006.430088.

29. C. O. Barnes, C. A. Jette, M. E. Abernathy, K. A. Dam, S. R. Esswein, H. B. Gristick, A. G. Malyutin, N. G. Sharaf, K. E. Huey-Tubman, Y. E. Lee, D. F. Robbiani, M. C. Nussenzweig, A. P. West, Jr. and P. J. Bjorkman, Nature, 2020, 588, 682-687.

30. W. Zhang, B. D. Davis, S. S. Chen, J. M. S. Martinez, J. T. Plummer and E. Vail, medRxiv, 2021, DOI: 10.1101/2021.01.18.21249786, 2021.2001.2018.21249786.

31. F. Bertoglio, V. Fühner, M. Ruschig, P. A. Heine, U. Rand, T. Klünemann, D. Meier, N. Langreder, S. Steinke, R. Ballmann, K.-T. Schneider, K. D. R. Roth, P. Kuhn, P. Riese, D. Schäckermann, J. Korn, A. Koch, S. Zock-Emmenthal, M. Becker, M. Scholz, G. M. S. G. Moreira, E. V. Wenzel, G. Russo, H. S. P. Garritsen, S. Casu, A. Gerstner, G. Roth, A. Hermann, T. Schirrmann, S. Dübel, A. Frenzel, J. Van den Heuvel, L. Čičin-Šain, M. Schubert and M. Hust, bioRxiv, 2020, DOI: 10.1101/2020.12.03.409318, 2020.2012.2003.409318.

32. S. Miersch, Z. Li, R. Saberianfar, M. Ustav, J. B. Case, L. Blazer, C. Chen, W. Ye, A. Pavlenco, M. Gorelik, J. G. Perez, S. Subramania, S. Singh, L. Ploder, S. Ganaie, R. E. Chen, D. W. Leung, P. P. Pandolfi, G. Novelli, G. Matusali, F. Colavita, M. R. Capobianchi, S. Jain, J. B. Gupta, G. K. Amarasinghe, M. S. Diamond, J. Rini and S. S. Sidhu, bioRxiv, 2020, DOI: 10.1101/2020.10.31.362848.

33. S. Du, Y. Cao, Q. Zhu, P. Yu, F. Qi, G. Wang, X. Du, L. Bao, W. Deng, H. Zhu, J. Liu, J. Nie, Y. Zheng, H. Liang, R. Liu, S. Gong, H. Xu, A. Yisimayi, Q. Lv, B. Wang, R. He, Y. Han, W. Zhao, Y. Bai, Y. Qu, X. Gao, C. Ji, Q. Wang, N. Gao, W. Huang, Y. Wang, X. S. Xie, X. D. Su, J. Xiao and C. Qin, Cell, 2020, 183, 1013-1023 e1013.

34. F. A. Tablizo, K. M. Kim, C. M. Lapid, M. J. R. Castro, M. S. L. Yangzon, B. A. Maralit, M. E. C. Ayes, E. M. Cutiongco-de la Paz, A. R. De Guzman, J. M. C. Yap, J.-H. S. Llames, S. M. M. Araiza, K. P. Punayan, I. C. A. Asin, C. F. B. Tambaoan, A. L. U. Chong, K. S. A. R. Padilla, R. P. S. Cruz, E. K. D. Morado, J. G. A. Dizon, R. N. M. Hao, A. A. Zamora, D. R. Pacial, J. A. R. Magalang, M. Alejandria, C. Carlos, A. Ong-Lim, E. M. Salvaña, J. Q. Wong, J. C. Montoya, M. R. Singh-Vergeire and C. P. Saloma, medRxiv, 2021, DOI: 10.1101/2021.03.03.21252812, 2021.2003.2003.21252812.

35. J. Schymkowitz, J. Borg, F. Stricher, R. Nys, F. Rousseau and L. Serrano, Nucleic Acids Res, 2005, 33, W382-388.

36. R. Guerois, J. E. Nielsen and L. Serrano, J Mol Biol, 2002, 320, 369-387. 


\section{Electronic Supplementary Information}

SARS-CoV-2 VOCs immune evasion from previously elicited neutralizing antibodies is mainly driven by lower cross-reactivity due to Spike RBD electrostatic surface changes

Matheus V. F. Ferraz ${ }^{a, b}$, Emerson G. Moreira ${ }^{a, b}$, Danilo F. Coêlho ${ }^{a, b}$, Gabriel Luz Wallau and Roberto D. Lins ${ }^{a,}$ on behalf of Fiocruz COVID-19 Genomic Surveillance Network

${ }^{a}$ Aggeu Magalhães Institute, Oswaldo Cruz Foundation, Recife, $P E$, Brazil

${ }^{b}$ Department of Fundamental Chemistry, Federal University of Pernambuco, Recife, PE, Brazil

*Corresponding author: roberto.lins@cpqam.fiocruz.br

1. Experimental values for $\Delta \Delta \mathrm{G}_{\text {Bind }}$ between RBD and hACE2 upon single mutations ........11

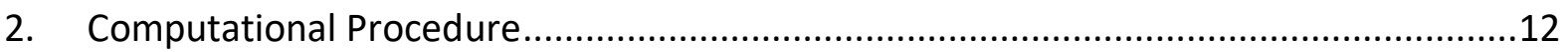

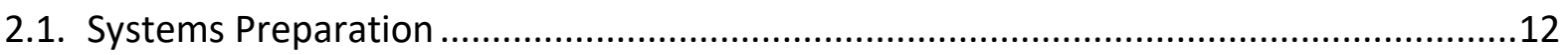

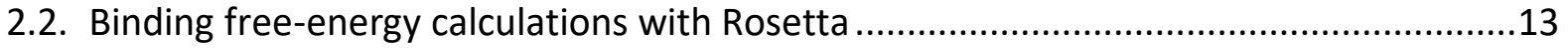

2.3. Command-lines and XML scripts for the calculations with the Rosetta package ...........15

2.3.1. . Rosetta Command-lines 15

2.3.2. RosettaScripts

15

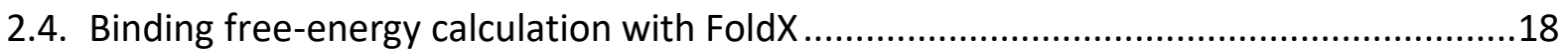

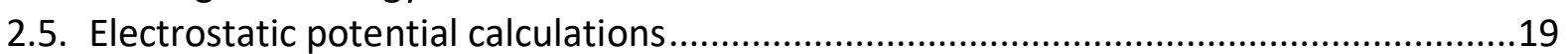

3. Antibodies dataset used for $\Delta \Delta \mathrm{G}_{\text {Bind }}$ computational calculations ...............................19

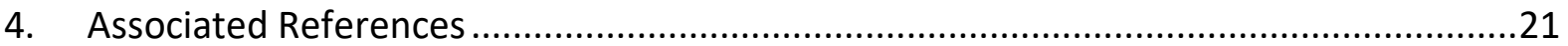




\section{Experimental values for $\Delta \Delta G_{\text {Bind }}$ between RBD and hACE2 upon single mutations}

Starr et al. ${ }^{1}$ obtained deep mutational scan data for all sites of interaction between SARS-CoV2 RBD and hACE2, transformed to values of $\triangle \log \left(K_{D}\right)$ according to the following relation:

$$
\operatorname{\Delta log}\left(K_{D}\right)=\log \left(K_{D, W T} \mid\right)-\log \left(K_{D, M U T} \mid\right) \quad \text { eq. } 1
$$

where $K_{D}$ is the dissociation constant and WT are values for wild-type and MUT for mutated variant. To transform the values into differences in binding free-energy, the following mathematical relationship was used:

$$
\begin{gathered}
\Delta \Delta G_{\text {Bind }}=\Delta G_{M U T}-\Delta G_{M U T}=R T\left[\ln \left(K_{D, M U T}\right)-\ln \left(K_{D, W T}\right)\right]=R T \ln \left(\frac{K_{D, M U T}}{K_{D, W T}}\right)= \\
-R T \ln \left(\frac{K_{D, W T}}{K_{D, M U T}}\right) \quad \text { eq. } 2
\end{gathered}
$$

From eq. 1 we have:

$$
\Delta \log \left(K_{D}\right)=\log \left(\frac{K_{D, W T}}{K_{D, M U T}}\right) \therefore \frac{K_{D, W T}}{K_{D, M U T}}=10^{\Delta \log \left(K_{D}\right)} \quad \text { eq. } 3
$$

Substituting eq. 3 in eq. 2 , we then have:

$$
\Delta \Delta G_{\text {Bind }}=-R T \ln \left(10^{\Delta \log \left(K_{D}\right)}\right)=-\Delta \log \left(K_{D}\right) \cdot R \operatorname{Rln}(10 \mid \quad) \quad \text { eq. } 4
$$

Using $\mathrm{T}=298 \mathrm{~K}\left(25 \stackrel{\circ}{ } \mathrm{C}\right.$ ) and $\mathrm{R}=1.987 \mathrm{kcal} \cdot \mathrm{K}^{-1} \cdot \mathrm{mol}^{-1}$ we transformed all $\Delta \log \left(K_{D}\right)$ experimental values into $\Delta \Delta G_{\text {Bind }}$ using eq. 4 . Figure S1 shows the heat map plot for the values. 


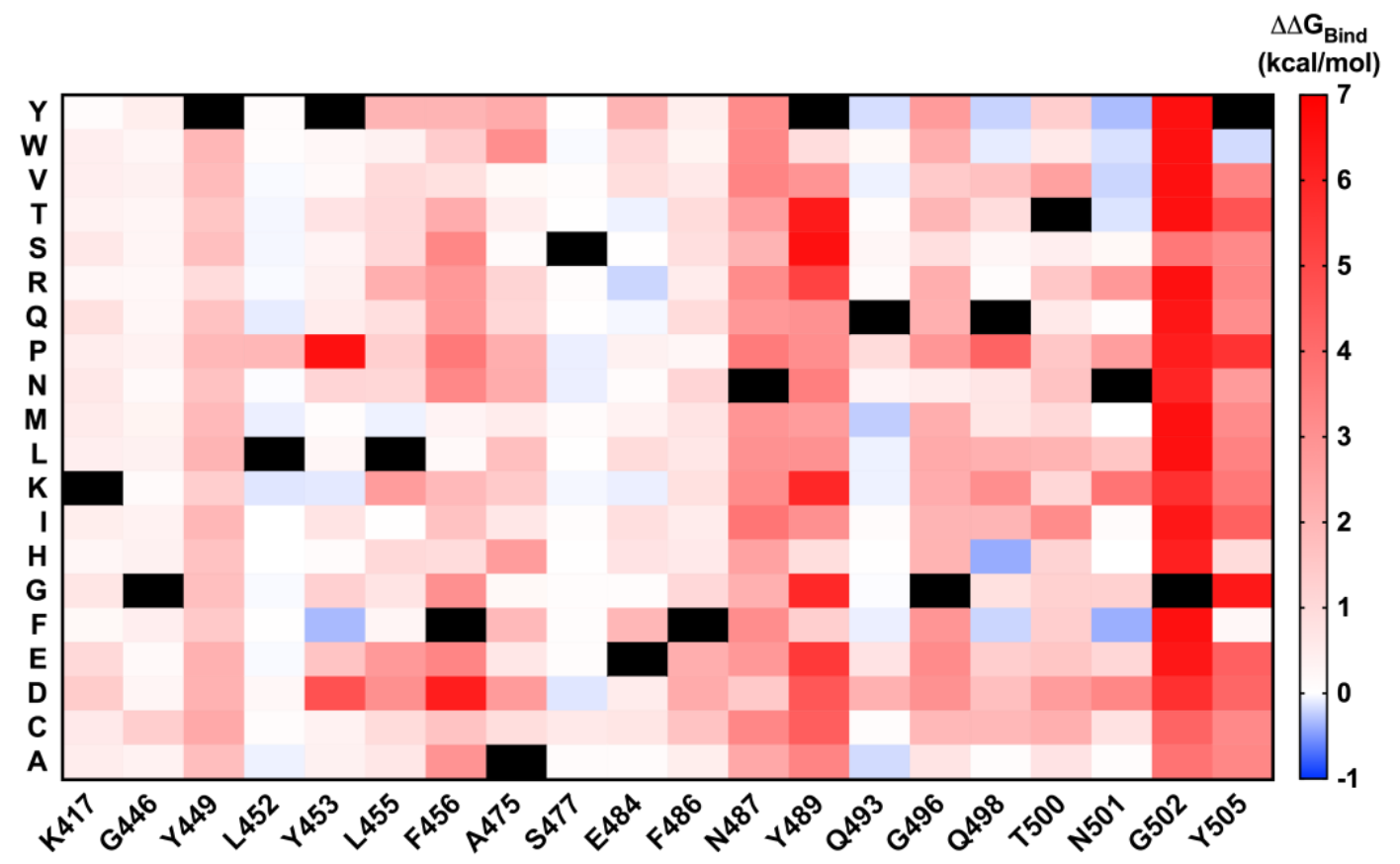

Figure S.1 - Heat map plot of Deep Mutational Scan $\Delta \Delta \boldsymbol{G}_{\boldsymbol{B} \text { ind }}$ values converted from experimental data retrieved from Starr et al. ${ }^{1}$ Horizontal-axis shows amino acid position in RBD and correspondent native amino acid. The vertical axis shows the identity of mutation amino acid. Values range from highly unfavorable changes (red squares) to marginally favorable change (blue), whereas black squares denote mutation to native amino acid (no experimental data available).

\section{Computational Procedure}

\subsection{Systems Preparation}

Atomic coordinates for the three-dimensional structure of the complex hACE2- SARS-CoV-2 RBD were retrieved from the Protein Data Bank (PDB) under accession code 6M0J. ${ }^{2} 27$ crystal structures of nAbs bound to SARS-Cov-2 RBD were downloaded from the PDB. The structures were cleaned by removing crystallographic water molecules, ligands, and ions. Only structures without missing residues in the interface between RBD and the respective antibody were considered, resulting in a total of 22 structures (accession code in Table S2). The missing residues lying far from the binding interface were modeled using Swiss-Model. ${ }^{3,4}$ Then, 4 steps of the FastRelax protocol, as implemented in the Rosetta package v. $3.12,{ }^{5}$ were used 
to pack and minimize the side-chains and backbone's conformations. Recognized VOCs (B.1.1.7, B.1.351 and P.1) and VOIs (P.2, B.1.429, B.1.526) with known epidemiological importance and bearing a number of key mutations in the RBD of the S protein (https://covlineages.org/ and https://www.cdc.gov/coronavirus/2019-ncov/cases-updates/variantsurveillance/variant-info.html) (Table S1) ${ }^{6}$ that were previously evaluated in experimental studies were recovered from the GISAID database (https://www.gisaid.org/) and used to estimate binding affinities with hACE2 and nAbs. Moreover, we also included a recent emerged variant ${ }^{7}$ (P.3 - https://cov-lineages.org/lineages/lineage_P.3.html) bearing additional Spike mutations to investigate its binding affinities to the hACE2 and nAbs as well.

Table S. 1 - Mutations in RBD of each VOC/VOI evaluated

\begin{tabular}{ccc}
\hline VOC/VOI & RBD mutation & GISAID ID \\
\hline B.1.351 & $\begin{array}{c}\text { K417N E484K } \\
\text { N501Y }\end{array}$ & EPI_ISL_968081 \\
\hline B.1.1.7 & N501Y & EPI_ISL_601443 \\
\hline P.1 & K417T E484K & EPI_ISL_833137 \\
N501Y & \\
\hline B.1.429 & L452R & EPI_ISL_1402625 \\
\hline B.1.526 & S477N E484K & EPI_ISL_1081256 \\
\hline P.2 & E484K & EPI_ISL_792560 \\
\hline P.3 & E484K N501Y & EPI_ISL_1122426
\end{tabular}

\subsection{Binding free-energy calculations with Rosetta}

To compute the change in binding free energy $(\Delta \Delta G)$ of hACE2-RBD and nAbs-RBD upon residues mutation, the Rosetta v.3.12 $2^{5}$ package was used. The Rosetta package is a prime-tool in protein modeling and design, ${ }^{8,9}$ and it has an empirical energy function ${ }^{10,11}$ that predicts mutational $\Delta \Delta G$ with a Pearson's correlation coefficient of 0.72 with mean absolute error (MAE) of $1.7 \mathrm{kcal}_{\mathrm{mol}}{ }^{-1}$, as tested on a dataset of 1240 mutations. ${ }^{12}$ The calculations were 
conducted using the scripting language interface RosettaScripts ${ }^{13,}{ }^{14}$ (code and parsed command-lines described below). The employed XML was adapted from a previously published script elsewhere. ${ }^{12}$ The employed energy function was the Talaris $2014 .{ }^{11}$ Initially, the wild-type structure was repacked using harmonic restraints on pairwise bond lengths. Then, the structure was minimized maintaining the bond lengths restraints using a maximum 5.000 iterations and tolerance of 0.001 REU by the Limited-memory Broyden-FletcherGoldfarb-Shanno algorithm complying with the Armijo-Goldstein condition, as implemented in the Rosetta package. For these steps a constraint term of weight of 1.0 was added to the score function. The constraints were released, and the wild-type binding free energy $\left(\Delta G_{W T}\right)$ was calculated by averaging over 10 repeats of the Ddg filter. Then, mutations were performed with the PackRotamersMover, and residues within a sphere of $8 \AA$ of the mutation(s) site(s) were repacked. The same harmonic restraints were used to the mutated structure and the same level of convergence was used to minimize the geometry of the mutant. Then, upon constraints release the mutant binding free energy ( $\Delta G_{M U T}$ ) was computed using the Ddg filter. The $\Delta \Delta \mathrm{G}$ was calculated as $\Delta \Delta \mathrm{G}=\Delta \mathrm{G}_{\mathrm{MUT}}-\Delta \mathrm{G}_{\mathrm{WT}}$. Prior to assessing the impact of the VOCs' mutations in the RBD to the $\Delta \Delta G$ towards the binding to hACE2, we have tested the performance of the Rosetta energy function to predict the $\Delta \Delta G$ for mutations contained in the dataset from the deep mutational scanning, comprising ca. 4,000 mutations. Thus, the $\Delta \Delta G$ for every single mutation was computed and compared to the experimental value as from the deep mutational scanning data. The performance of the Rosetta-predicted values for the $\Delta \Delta G$ was measured using the $\mathrm{MAE}$, calculated according to eq. 5 , as follow:

$$
M A E=\frac{\sum_{i=1}^{n} y_{i}-\bar{y}_{i} \vee}{n} \quad \text { eq. } 5
$$

Where $\mathrm{n}$ consists of the total sample size, $y_{i}$ is the predicted $\Delta \Delta G$ from rosetta calculations, and $\bar{y}_{i}$ is the experimentally observed value for $\Delta \Delta G$ from the deep mutational scanning data. 


\subsection{Command-lines and XML scripts for the calculations with the Rosetta package}

\subsubsection{Rosetta Command-lines}

The following command-lines were parsed:

Relax the PDB: \$rosetta/main/source/bin/rosetta_scripts.macosgccrelease -database rosetta/main/database/ -s *.pdb -parser:protocol relax.xml -ex1 -ex2 -use_input_sc

Binding free-energy evaluation:

\$rosetta/main/source/bin/rosetta_scripts.macosgccrelease -database rosetta/main/database/ $\quad$-s $\quad *$.pdb -parser:protocol ddg.xml -parser:script_vars file=mutation*.resfile -ex1 -ex2 -use_input_sc -restore_talaris_behavior

\subsubsection{RosettaScripts}

Relax (relax.xml)

$<$ ROSETTASCRIPTS $>$

$<$ MOVERS >

$<$ FastRelax name="fstrlx" repeats="4"/>

$</$ MOVERS $>$

$<$ FILTERS $>$

<Geometry name="omega" omega="150" cart_bonded="100" confidence="0" />

$<$ Rmsd name="rmsd" confidence="0" superimpose="1"/>

$</$ FILTERS $>$

<PROTOCOLS>

$<$ Add filter_name="omega"/>

$<$ Add mover_name="fstrlx"/>

$<$ Add filter_name="rmsd"/>

$</$ PROTOCOLS $>$

$</$ ROSETTASCRIPTS $>$ 
Binding free-energy evaluation (ddg.xml)

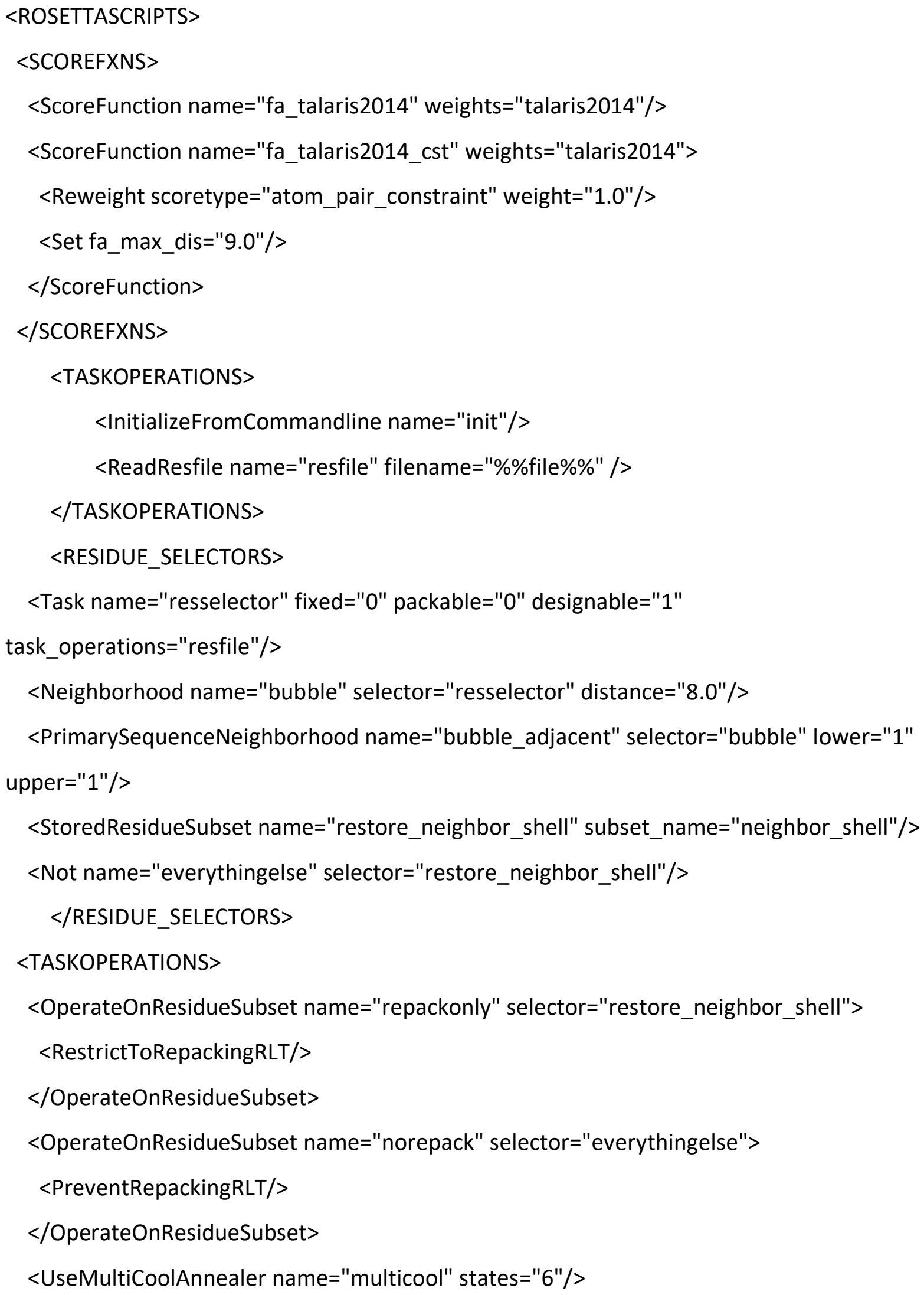




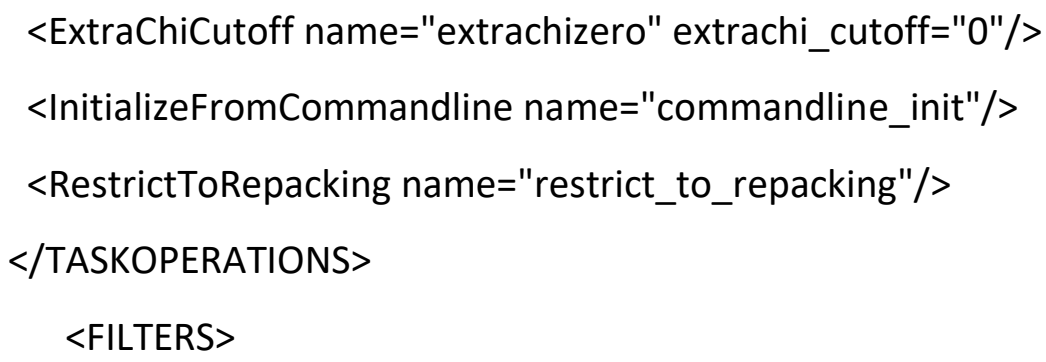




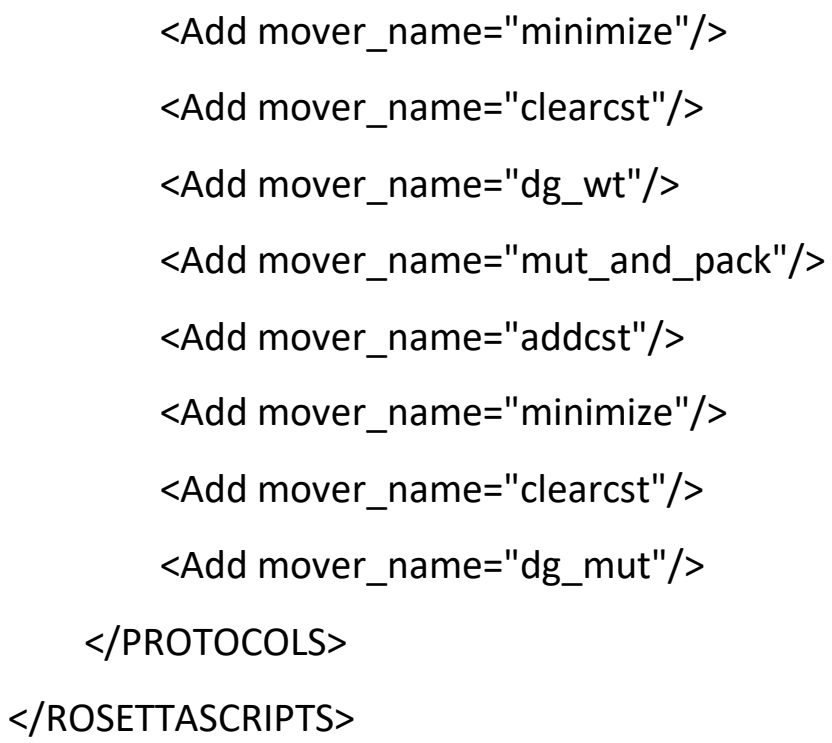

\subsection{Binding free-energy calculation with FoldX}

For the calculations using FoldX, the relaxed input PDBs were geometry-optimized using the FoldX force field and the RepairPDB command. The binding energy of the wild-type complex $\left(\Delta G_{W T}\right)$ was calculated using the AnalyseComplex command and was assumed as being corresponding to the interaction energy term. Then, mutations in the RBD for the different variants were introduced using a single run of the BuildModel. The binding energy of the mutated complex ( $\Delta \mathrm{G}_{\mathrm{MUT}}$ ) was calculated with the AnalyseComplex, and the $\Delta \Delta \mathrm{G}_{\text {Bind }}$ was taken as $\Delta \Delta G_{\text {Bind }}=\left(\Delta G_{M U T}-\Delta G_{W T}\right)$. For all FoldX calculations, the $p H$ was set as 7.0 and a solution ionic strength of $0.05 \mathrm{M}$. The full command-lines were as following:

Repair PDB: \$foldx $\quad$--command=RepairPDB $\quad--\mathrm{pdb}=*$.pdb $\quad$--ionStrength=0.05 $\quad$-- $\mathrm{pH}=7 \quad$-vdwDesign=2 --pdbHydrogens=false - water=predict

Mutation: \$foldx --command=BuildModel --pdb=*Repaired.pdb --mutantfile=mutations_list.txt --ionStrength=0.05 --pH=7 --water=predict --vdwDesign=2 --pdbHydrogens=false

Binding free-energy evaluation: \$foldx --command=AnalyseComplex --analyseComplexChains=A,E --pdb=*.pdb 
Figure $S 2$ below shows the $\Delta \Delta G_{\text {Bind }}$ for the complexes hACE2-RBD and RBD-nAbs upon mutations of each lineage using FoldX force-field:

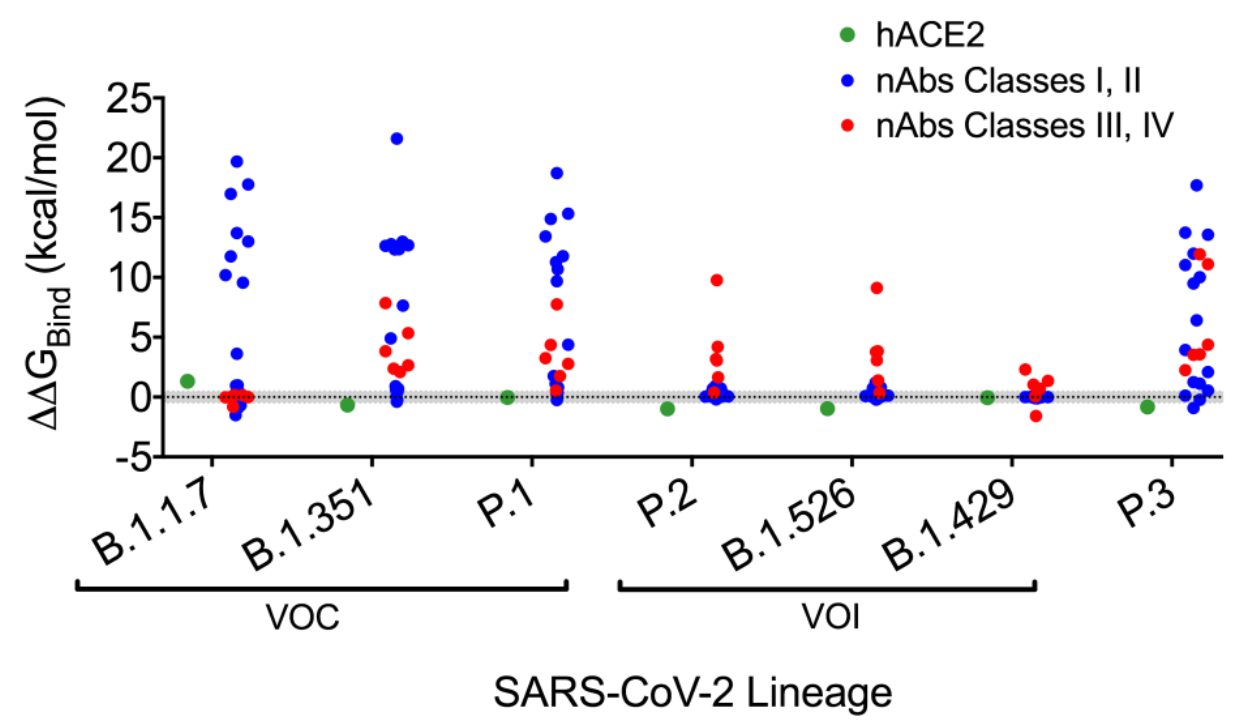

Figure S.2 - Variation in the binding free-energy upon mutation of each lineage for the hACE2RBD and nAbs-RBD complexes computed using FoldX. The hatched area indicates the MAE of the FoldX energy function centered at 0 .

\subsection{Electrostatic potential calculations}

Surface electrostatic potentials were plotted for the RBDs (wild-type and variants) and rendered utilizing the PyMol software (The PyMOL Molecular Graphics System, Version 2.0 Schrödinger, $\mathrm{LLC})$. PDB2PQR ${ }^{15}$ was used to generate pqr files by assigning partial atomic charges for the protein from the AMBER ff99 all-atom force field ${ }^{16}$ at $\mathrm{pH}=7$ with the solvent described by a dielectric constant of 78 and saline $(\mathrm{NaCl})$ concentration of $0.15 \mathrm{M}$ with ions of radius $1.5 \AA$. The low-dielectric cavity $(\varepsilon r)$ was assumed as 4 , using Bondi atomic radii. The linearized Poisson-Boltzmann equation (LPBE) was solved using the APBS code. ${ }^{17}$

\section{Antibodies dataset used for $\Delta \Delta \mathrm{G}_{\text {Bind }}$ computational calculations}

All PDB files were downloaded from RSC Protein Data Bank (https://www.rcsb.org/) and only structures determined by means of X-Ray crystallography and RBD with $100 \%$ identity to Wuhan lineage were used. Table S2 shows the structures dataset. 
Table S2. Antibodies dataset used for computational calculations

$\Delta \Delta G_{\text {Bind }} *$

\begin{tabular}{|c|c|c|c|c|c|c|c|c|c|}
\hline PDB ID & nAb Name & $\begin{array}{c}\text { nAb } \\
\text { Class }^{\dagger}\end{array}$ & B.1.351 & B.1.1.7 & P.1 & B.1.429 & B.1.526 & P.2 & P.3 \\
\hline $6 \times C 4$ & CC12.3 & I, II & $\begin{array}{c}0.25 \\
(0.92) \\
\end{array}$ & $\begin{array}{l}-0.59 \\
(0.35) \\
\end{array}$ & $\begin{array}{c}-0.51 \\
(1.11) \\
\end{array}$ & $\begin{array}{l}-0.55 \\
(0.01) \\
\end{array}$ & $\begin{array}{c}-2.9 \\
(0.09) \\
\end{array}$ & $\begin{array}{c}-1.6 \\
(0.05) \\
\end{array}$ & $\begin{array}{l}-0.419 \\
(1.12) \\
\end{array}$ \\
\hline 6XKQ & CV07-250 & I, II & $\begin{array}{c}0.6 \\
(-0.24) \\
\end{array}$ & $\begin{array}{l}-0.95 \\
(0.99) \\
\end{array}$ & $\begin{array}{c}0.06 \\
(-0.25) \\
\end{array}$ & $\begin{array}{l}-0.83 \\
(0.02) \\
\end{array}$ & $\begin{array}{c}0.39 \\
(0.13) \\
\end{array}$ & $\begin{array}{l}-0.96 \\
(0.39) \\
\end{array}$ & $\begin{array}{l}-0.49 \\
(2.1) \\
\end{array}$ \\
\hline 7B30 & STE90-C11 & I, II & $\begin{array}{c}16.85 \\
(12.33) \\
\end{array}$ & $\begin{array}{c}15.8 \\
(16.98) \\
\end{array}$ & $\begin{array}{l}15.57 \\
(9.68) \\
\end{array}$ & $\begin{array}{l}-0.45 \\
(0.02) \\
\end{array}$ & $\begin{array}{l}-0.52 \\
(0.01) \\
\end{array}$ & $\begin{array}{l}-0.76 \\
(0.03) \\
\end{array}$ & $\begin{array}{l}15.65 \\
(9.49) \\
\end{array}$ \\
\hline 7BEI & covox-150 & I, II & $\begin{array}{c}15.99 \\
(12.63) \\
\end{array}$ & $\begin{array}{c}15.26 \\
(11.76) \\
\end{array}$ & $\begin{array}{c}16.5 \\
(10.69)\end{array}$ & $\begin{array}{c}-0.23 \\
(-0.02) \\
\end{array}$ & $\begin{array}{c}0.45 \\
(0.81) \\
\end{array}$ & $\begin{array}{c}0.46 \\
(0.71) \\
\end{array}$ & $\begin{array}{c}15.84 \\
(11.98) \\
\end{array}$ \\
\hline 7BEJ & $\begin{array}{c}\text { COVOX- } \\
158^{\&}\end{array}$ & I, II & $\begin{array}{c}6.79 \\
(7.64) \\
\end{array}$ & $\begin{array}{c}6.36 \\
(10.19) \\
\end{array}$ & $\begin{array}{c}6.71 \\
(11.29) \\
\end{array}$ & $\begin{array}{l}-0.25 \\
(0.01) \\
\end{array}$ & $\begin{array}{l}-0.23 \\
(0.15) \\
\end{array}$ & $\begin{array}{c}-0.12 \\
(-0.03) \\
\end{array}$ & $\begin{array}{c}6.26 \\
(6.41) \\
\end{array}$ \\
\hline 7BEK & $\begin{array}{c}\text { Covox- } \\
158^{\&}\end{array}$ & I, II & $\begin{array}{c}14.16 \\
(12.77) \\
\end{array}$ & $\begin{array}{c}14.96 \\
(13.01)\end{array}$ & $\begin{array}{c}13.65 \\
(14.89) \\
\end{array}$ & $\begin{array}{c}0.08 \\
(0.01) \\
\end{array}$ & $\begin{array}{l}-0.01 \\
(0.03) \\
\end{array}$ & $\begin{array}{l}-0.01 \\
(0.03) \\
\end{array}$ & $\begin{array}{c}15.17 \\
(13.75) \\
\end{array}$ \\
\hline 7BEM & COVOX-269 & I, II & $\begin{array}{c}14.53 \\
(12.69) \\
\end{array}$ & $\begin{array}{c}12 \\
(13.71) \\
\end{array}$ & $\begin{array}{c}14.74 \\
(13.42) \\
\end{array}$ & $\begin{array}{c}-1.1 \\
(-0.10) \\
\end{array}$ & $\begin{array}{c}-0.63 \\
(-0.01) \\
\end{array}$ & $\begin{array}{l}-1.26 \\
(0.02) \\
\end{array}$ & $\begin{array}{c}11.92 \\
(11.03) \\
\end{array}$ \\
\hline 7C01 & CB6 & I, II & $\begin{array}{c}1.54 \\
(0.71) \\
\end{array}$ & $\begin{array}{c}-1.8 \\
(-0.89) \\
\end{array}$ & $\begin{array}{c}1.07 \\
(1.75) \\
\end{array}$ & $\begin{array}{l}-1.02 \\
(0.08) \\
\end{array}$ & $\begin{array}{l}-0.25 \\
(-0.21) \\
\end{array}$ & $\begin{array}{l}-0.98 \\
(-0.2) \\
\end{array}$ & $\begin{array}{c}-1.72 \\
(-0.91) \\
\end{array}$ \\
\hline $7 \mathrm{CH} 4$ & BD-604 & I, II & $\begin{array}{c}12.16 \\
(12.99) \\
\end{array}$ & $\begin{array}{c}9.11 \\
(19.69)\end{array}$ & $\begin{array}{c}10.84 \\
(15.32)\end{array}$ & $\begin{array}{c}-0.35 \\
(-0.02) \\
\end{array}$ & $\begin{array}{c}0.11 \\
(-0.05)\end{array}$ & $\begin{array}{l}-0.45 \\
(-0.03)\end{array}$ & $\begin{array}{c}8.93 \\
(13.56)\end{array}$ \\
\hline $7 \mathrm{CH} 5$ & BD-629 & I, II & $\begin{array}{c}1.41 \\
(0.06) \\
\end{array}$ & $\begin{array}{c}1.05 \\
(0.97) \\
\end{array}$ & $\begin{array}{c}1.29 \\
(-0.13) \\
\end{array}$ & $\begin{array}{c}-0.35 \\
(0) \\
\end{array}$ & $\begin{array}{l}-0.94 \\
(0.15) \\
\end{array}$ & $\begin{array}{l}-0.07 \\
(0.35) \\
\end{array}$ & $\begin{array}{c}1.08 \\
(1.24) \\
\end{array}$ \\
\hline $7 \mathrm{CHB}$ & BD-236 & I, II & $\begin{array}{c}21.79 \\
(12.35) \\
\end{array}$ & $\begin{array}{l}17.39 \\
(9.56) \\
\end{array}$ & $\begin{array}{c}20.68 \\
(11.77) \\
\end{array}$ & $\begin{array}{c}-0.96 \\
(-0.09) \\
\end{array}$ & $\begin{array}{c}-0.56 \\
(-0.03) \\
\end{array}$ & $\begin{array}{c}-0.47 \\
(-0.01) \\
\end{array}$ & $\begin{array}{c}17.4 \\
(10.01) \\
\end{array}$ \\
\hline $7 \mathrm{~K} 8 \mathrm{M}$ & C102 & I, II & $\begin{array}{c}6.86 \\
(21.6) \\
\end{array}$ & $\begin{array}{c}15.25 \\
(17.76) \\
\end{array}$ & $\begin{array}{c}14.43 \\
(18.71) \\
\end{array}$ & $\begin{array}{l}-0.03 \\
(0.02) \\
\end{array}$ & $\begin{array}{c}0.67 \\
(-0.16) \\
\end{array}$ & $\begin{array}{c}-0.66 \\
(-0.06) \\
\end{array}$ & $\begin{array}{l}15.31 \\
(17.7) \\
\end{array}$ \\
\hline $7 K L G$ & 15033 & I, II & $\begin{array}{c}1.03 \\
(0.49) \\
\end{array}$ & $\begin{array}{c}-0.94 \\
(-0.69) \\
\end{array}$ & $\begin{array}{c}2.97 \\
(0.37) \\
\end{array}$ & $\begin{array}{c}-0.64 \\
(-0.01) \\
\end{array}$ & $\begin{array}{l}2.742 \\
(0.74) \\
\end{array}$ & $\begin{array}{c}2.99 \\
(0.72) \\
\end{array}$ & $\begin{array}{c}3.13 \\
(0.12) \\
\end{array}$ \\
\hline 7KLH & $15033-7$ & I, II & $\begin{array}{c}4.5 \\
(0.62) \\
\end{array}$ & $\begin{array}{c}0.3 \\
(-1.51) \\
\end{array}$ & $\begin{array}{c}3.8 \\
(0.8) \\
\end{array}$ & $\begin{array}{c}-0.4 \\
(-0.03) \\
\end{array}$ & $\begin{array}{c}2.3 \\
(1.22) \\
\end{array}$ & $\begin{array}{c}2.6 \\
(0.94) \\
\end{array}$ & $\begin{array}{c}2.68 \\
(-0.22) \\
\end{array}$ \\
\hline 7CDI & P2C-1F11 & I, II & $\begin{array}{c}0.96 \\
(4.91) \\
\end{array}$ & $\begin{array}{l}-0.23 \\
(3.62) \\
\end{array}$ & $\begin{array}{c}0.83 \\
(4.37) \\
\end{array}$ & $\begin{array}{c}-0.28 \\
(-0.04) \\
\end{array}$ & $\begin{array}{l}0.25 \\
(0.3) \\
\end{array}$ & $\begin{array}{c}-0.21 \\
(0.32) \\
\end{array}$ & $\begin{array}{c}0.04 \\
(3.93) \\
\end{array}$ \\
\hline 7BZ5 & B38 & I, II & $\begin{array}{c}0.79 \\
(-0.39) \\
\end{array}$ & $\begin{array}{l}-0.59 \\
(0.52) \\
\end{array}$ & $\begin{array}{c}0.85 \\
(0.29) \\
\end{array}$ & $\begin{array}{c}-1.25 \\
(0) \\
\end{array}$ & $\begin{array}{c}-0.94 \\
(0.01) \\
\end{array}$ & $\begin{array}{l}-0.58 \\
(0.02) \\
\end{array}$ & $\begin{array}{l}-0.88 \\
(0.55) \\
\end{array}$ \\
\hline $6 X K P$ & CV07-270 & III, IV & $\begin{array}{c}4.72 \\
(3.84) \\
\end{array}$ & $\begin{array}{c}-1.32 \\
(0) \\
\end{array}$ & $\begin{array}{c}4.31 \\
(3.26) \\
\end{array}$ & $\begin{array}{c}4.2 \\
(2.3) \\
\end{array}$ & $\begin{array}{c}4.51 \\
(3.84) \\
\end{array}$ & $\begin{array}{c}4.67 \\
(3.18) \\
\end{array}$ & $\begin{array}{c}4.55 \\
(3.56) \\
\end{array}$ \\
\hline 7BEH & covox-316 & III, IV & $\begin{array}{c}8.45 \\
(2.38) \\
\end{array}$ & $\begin{array}{l}-0.55 \\
(-0.8) \\
\end{array}$ & $\begin{array}{c}8.59 \\
(2.78) \\
\end{array}$ & $\begin{array}{l}-1.49 \\
(0.07) \\
\end{array}$ & $\begin{array}{c}8.46 \\
(3.08) \\
\end{array}$ & $\begin{array}{c}8.2 \\
(3.05) \\
\end{array}$ & $\begin{array}{c}8.34 \\
(2.25) \\
\end{array}$ \\
\hline 7BWJ & P2B-2F6 & III, IV & $\begin{array}{l}6.29 \\
(2.1) \\
\end{array}$ & $\begin{array}{c}-0.9 \\
(0) \\
\end{array}$ & $\begin{array}{c}0.09 \\
(1.75) \\
\end{array}$ & $\begin{array}{l}-1.11 \\
(1.04) \\
\end{array}$ & $\begin{array}{c}0.07 \\
(1.38) \\
\end{array}$ & $\begin{array}{c}0.17 \\
(1.65) \\
\end{array}$ & $\begin{array}{c}0.02 \\
(11.1) \\
\end{array}$ \\
\hline 7L5B & $2-15$ & III, IV & $\begin{array}{c}6.92 \\
(2.66)\end{array}$ & $\begin{array}{l}-2.59 \\
(0.16)\end{array}$ & $\begin{array}{c}6.37 \\
(0.55)\end{array}$ & $\begin{array}{c}-1.2 \\
(0.7)\end{array}$ & $\begin{array}{c}7.77 \\
(0.35)\end{array}$ & $\begin{array}{c}7.61 \\
(0.37)\end{array}$ & $\begin{array}{c}6.08 \\
(3.53)\end{array}$ \\
\hline
\end{tabular}




\begin{tabular}{|c|c|c|c|c|c|c|c|c|c|}
\hline 7KMG & LY-CoV555 & III, IV & $\begin{array}{l}11.55 \\
(7.85)\end{array}$ & $\begin{array}{c}-0.54 \\
(-0.02) \\
\end{array}$ & $\begin{array}{l}11.54 \\
(7.75)\end{array}$ & $\begin{array}{c}2.32 \\
(1.35)\end{array}$ & $\begin{array}{c}11.4 \\
(9.13)\end{array}$ & $\begin{array}{l}14.35 \\
(9.78)\end{array}$ & $\begin{array}{c}11.54 \\
(11.94) \\
\end{array}$ \\
\hline 7CDJ & $\mathrm{P} 2 \mathrm{C}-1 \mathrm{~A} 3$ & III, IV & $\begin{array}{c}9.7 \\
(5.35)\end{array}$ & $\begin{array}{c}-1.65 \\
(0.18) \\
\end{array}$ & $\begin{array}{l}12.54 \\
(4.36)\end{array}$ & $\begin{array}{c}-3.35 \\
(-1.58) \\
\end{array}$ & $\begin{array}{c}9.83 \\
(3.78) \\
\end{array}$ & $\begin{array}{l}9.88 \\
(4.2) \\
\end{array}$ & $\begin{array}{c}9.6 \\
(4.38)\end{array}$ \\
\hline
\end{tabular}

* Values outside parenthesis are for Rosetta (REU units); values inside parenthesis are for Foldx $\left(\mathrm{kcal}^{\mathrm{mol}} \mathrm{m}^{-1}\right)$.

† According to Barnes et al. ${ }^{18}$ classes I and II comprise nAbs with epitopes that overlap with hACE2 binding site (class I binds Up/Down conformations, class II binds Up only); classes III and IV epitopes do not overlap with hACE2 binding site (class III binds Up conformation only, class IV binds Up/Down conformations). Since all PDBs evaluated in this work are for nAbs complexed with isolated RBD domain, the classifications were based by aligning to hACE2RBD complex (PDB: 6MOJ) and evaluating if the epitopes overlap with hACE2 binding site.

\& The same $n A b$ is represented in two slightly different forms of binding to RBD.

\section{Associated References}

1. T. N. Starr, A. J. Greaney, S. K. Hilton, D. Ellis, K. H. D. Crawford, A. S. Dingens, M. J. Navarro, J. E. Bowen, M. A. Tortorici, A. C. Walls, N. P. King, D. Veesler and J. D. Bloom, Cell, 2020, 182, 1295.

2. J. Lan, J. Ge, J. Yu, S. Shan, H. Zhou, S. Fan, Q. Zhang, X. Shi, Q. Wang, L. Zhang and X. Wang, Nature, 2020, 581, 215.

3. N. Guex and M. C. Peitsch, Electrophoresis, 1997, 18, 2714.

4. A. Waterhouse, M. Bertoni, S. Bienert, G. Studer, G. Tauriello, R. Gumienny, F. T. Heer, T. A. P. de Beer, C. Rempfer, L. Bordoli, R. Lepore and T. Schwede, Nucleic Acids Res, 2018, 46, W296.

5. A. Leaver-Fay, M. Tyka, S. M. Lewis, O. F. Lange, J. Thompson, R. Jacak, K. Kaufman, P. D. Renfrew, C. A. Smith, W. Sheffler, I. W. Davis, S. Cooper, A. Treuille, D. J. Mandell, F. Richter, Y. E. Ban, S. J. Fleishman, J. E. Corn, D. E. Kim, S. Lyskov, M. Berrondo, S. Mentzer, Z. Popovic, J. J. Havranek, J. Karanicolas, R. Das, J. Meiler, T. Kortemme, J. J. Gray, B. Kuhlman, D. Baker and P. Bradley, Methods Enzymol, 2011, 487, 545.

6. C. M. Voloch, R. da Silva Francisco, L. G. P. de Almeida, C. C. Cardoso, O. J. Brustolini, A. L. Gerber, A. P. d. C. Guimarães, D. Mariani, R. M. da Costa, O. C. Ferreira, T. S. Frauches, C. M. B. de Mello, I. d. C. Leitão, R. M. Galliez, D. S. Faffe, T. M. P. P. Castiñeiras, A. Tanuri and A. T. R. de Vasconcelos, Journal of Virology, 2021, DOI: 10.1128/JVI.00119-21, JVI.00119.

7. F. A. Tablizo, K. M. Kim, C. M. Lapid, M. J. R. Castro, M. S. L. Yangzon, B. A. Maralit, M. E. C. Ayes, E. M. Cutiongco-de la Paz, A. R. De Guzman, J. M. C. Yap, J.-H. S. Llames, S. M. M. Araiza, K. P. Punayan, I. C. A. Asin, C. F. B. Tambaoan, A. L. U. Chong, K. S. A. R. Padilla, R. P. S. Cruz, E. K. D. Morado, J. G. A. Dizon, R. N. M. Hao, A. A. Zamora, D. R. Pacial, J. A. R. Magalang, M. Alejandria, C. Carlos, A. Ong-Lim, E. M. Salvaña, J. Q. Wong, J. C. Montoya, M. R. Singh-Vergeire and C. P. Saloma, medRxiv, 2021, DOI: 10.1101/2021.03.03.21252812, 2021.03.03.21252812. 
8. K. W. Kaufmann, G. H. Lemmon, S. L. Deluca, J. H. Sheehan and J. Meiler, Biochemistry, 2010, 49, 2987.

9. J. K. Leman, B. D. Weitzner, S. M. Lewis, J. Adolf-Bryfogle, N. Alam, R. F. Alford, M. Aprahamian, D. Baker, K. A. Barlow, P. Barth, B. Basanta, B. J. Bender, K. Blacklock, J. Bonet, S. E. Boyken, P. Bradley, C. Bystroff, P. Conway, S. Cooper, B. E. Correia, B. Coventry, R. Das, R. M. De Jong, F. DiMaio, L. Dsilva, R. Dunbrack, A. S. Ford, B. Frenz, D. Y. Fu, C. Geniesse, L. Goldschmidt, R. Gowthaman, J. J. Gray, D. Gront, S. Guffy, S. Horowitz, P. S. Huang, T. Huber, T. M. Jacobs, J. R. Jeliazkov, D. K. Johnson, K. Kappel, J. Karanicolas, H. Khakzad, K. R. Khar, S. D. Khare, F. Khatib, A. Khramushin, I. C. King, R. Kleffner, B. Koepnick, T. Kortemme, G. Kuenze, B. Kuhlman, D. Kuroda, J. W. Labonte, J. K. Lai, G. Lapidoth, A. Leaver-Fay, S. Lindert, T. Linsky, N. London, J. H. Lubin, S. Lyskov, J. Maguire, L. Malmstrom, E. Marcos, O. Marcu, N. A. Marze, J. Meiler, R. Moretti, V. K. Mulligan, S. Nerli, C. Norn, S. O'Conchuir, N. Ollikainen, S. Ovchinnikov, M. S. Pacella, X. Pan, H. Park, R. E. Pavlovicz, M. Pethe, B. G. Pierce, K. B. Pilla, B. Raveh, P. D. Renfrew, S. S. R. Burman, A. Rubenstein, M. F. Sauer, A. Scheck, W. Schief, O. Schueler-Furman, Y. Sedan, A. M. Sevy, N. G. Sgourakis, L. Shi, J. B. Siegel, D. A. Silva, S. Smith, Y. Song, A. Stein, M. Szegedy, F. D. Teets, S. B. Thyme, R. Y. Wang, A. Watkins, L. Zimmerman and R. Bonneau, Nat Methods, 2020, 17, 665.

10. R. F. Alford, A. Leaver-Fay, J. R. Jeliazkov, M. J. O'Meara, F. P. DiMaio, H. Park, M. V. Shapovalov, P. D. Renfrew, V. K. Mulligan, K. Kappel, J. W. Labonte, M. S. Pacella, R. Bonneau, P. Bradley, R. L. Dunbrack, Jr., R. Das, D. Baker, B. Kuhlman, T. Kortemme and J. J. Gray, J Chem Theory Comput, 2017, 13, 3031.

11. A. Bazzoli, S. P. Kelow and J. Karanicolas, PLoS One, 2015, 10, e0140359.

12. K. A. Barlow, O. C. S, S. Thompson, P. Suresh, J. E. Lucas, M. Heinonen and T. Kortemme, J Phys Chem B, 2018, 122, 5389.

13. S. J. Fleishman, A. Leaver-Fay, J. E. Corn, E. M. Strauch, S. D. Khare, N. Koga, J. Ashworth, P. Murphy, F. Richter, G. Lemmon, J. Meiler and D. Baker, PLoS One, 2011, 6, e20161.

14. B. J. Bender, A. Cisneros, 3rd, A. M. Duran, J. A. Finn, D. Fu, A. D. Lokits, B. K. Mueller, A. K. Sangha, M. F. Sauer, A. M. Sevy, G. Sliwoski, J. H. Sheehan, F. DiMaio, J. Meiler and R. Moretti, Biochemistry, 2016, 55, 4748.

15. T. J. Dolinsky, J. E. Nielsen, J. A. McCammon and N. A. Baker, Nucleic Acids Res, 2004, 32, W665.

16. A. Spasic, J. Serafini and D. H. Mathews, J Chem Theory Comput, 2012, 8, 2497.

17. N. A. Baker, D. Sept, S. Joseph, M. J. Holst and J. A. McCammon, Proc Natl Acad Sci U $S A, 2001,98,10037$.

18. C. O. Barnes, C. A. Jette, M. E. Abernathy, K.-M. A. Dam, S. R. Esswein, H. B. Gristick, A. G. Malyutin, N. G. Sharaf, K. E. Huey-Tubman, Y. E. Lee, D. F. Robbiani, M. C. Nussenzweig, A. P. West and P. J. Bjorkman, Nature, 2020, 588, 682. 